\title{
Vibrotactile Feedback as an Orientation Aid for Blind Users of Mobile Guides
}

\author{
Giuseppe Ghiani Barbara Leporini Fabio Paternò \\ ISTI-CNR \\ Via G. Moruzzi, 1 \\ 56124 Pisa, Italy \\ +390503153066
}

\{giuseppe.ghiani, barbara.leporini, fabio.paterno\}@isti.cnr.it

\begin{abstract}
In this paper, we present a solution for supporting vibrotactile feedback in mobile museum guides for blind users. To this end, we have designed and implemented a hardware/software module, which can be easily plugged into current PDAs to assist blind users in orientation. The solution, which comprises a twochannels haptic module as well as vocal support, has been exploited for moving through tagged objects. We also report on a user evaluation carried out with a number of blind users.
\end{abstract}

\section{Categories and Subject Descriptors}

H5.m. Information interfaces and presentation (e.g., HCI).

\section{General Terms}

Design, Experimentation, Human Factors

\section{INTRODUCTION}

Multimodal digital guides are increasingly used to support people during museum visits. In general, a digital guide allows people to visit the museum according to their preferences. For this reason, it is important that the guide be information-rich and at the same time adaptable to users' needs. When blind people visit a museum, usually they count on a person who accompanies them and describes the artworks or specimens visited. Thus, in order to have a pleasant visit the accompanying person should have appropriate skills especially in describing object details. In this perspective, a digital, accessible and well-designed guide can represent valuable support for a museum visit by blind people in an autonomous way. Moreover, the fact that blind people can make a visit autonomously can represent a good way to integrate them in a visitor group (e.g. family or friends). In fact, really participating in a museum visit is more effective than obtaining cultural information from a Web site or multimedia CD. In addition, such an opportunity represents a way to increase social inclusion as well. During the visit blind and sighted people can discuss and exchange opinions and comments on the nearby

Permission to make digital or hard copies of all or part of this work for personal or classroom use is granted without fee provided that copies are not made or distributed for profit or commercial advantage and that copies bear this notice and the full citation on the first page. To copy otherwise, or republish, to post on servers or to redistribute to lists, requires prior specific permission and/or a fee.

MobileHCI 2008, September 2-5, 2008, Amsterdam, the Netherlands.

Copyright @ 2008 ACM 978-1-59593-952-4/08/09...\$5.00. artworks. This should also improve the learning process. For all such reasons, a digital guide not only should provide useful information on the artworks or specimens, but it should also provide a certain support to assist the user when moving through the museum among the artworks.

While a considerable amount of research work has been dedicated to mobile guides, little attention has been paid to identify solutions accessible for the blind. To address such needs, we have carried out research aiming to design and implement a multimodal mobile guide, which is able to assist blind users when freely moving around the museum. In particular, in this paper we propose a multimodal and location-aware museum guide, which has been specifically designed for visually impaired people to provide them with flexible orientation support as well as with the speech description of visited sections/artworks. In our first trials we considered solutions based on vocal messages or sounds, which have already been tested with a group of blind users [7]. Such first tests provided encouraging feedback and also useful suggestions for considering additional modalities for providing orientation-related feedback. In particular, some users suggested also considering vibrotactile feedback. In this paper, after discussion of related work, we present the tactile module, which we have designed and integrated in our mobile guide to provide orientation-related feedback. Then, we report on a user test with blind users and provide some concluding remarks along with indications for future work.

\section{RELATED WORK}

The use of haptic interfaces for providing information nonvisually has been widely investigated. A study on how vibrotactile messages, called Tactons, can deliver complex information is presented in [3]. Authors focused on the use of tactile feedback alone and evaluated the delivery of structured messages by 3 tactile parameters (Rhythm, Roughness and Spatial Location) through several vibrotactile actuators. A wearable vibrotactile display made up of an array of 9 tactile actuators was tested in [10]. The previously cited studies focused on the use of tactile feedback alone (without audio) and were limited to stationary devices. Tactile output supporting mobile interactions was instead investigated in [2]. The experiments regarded text insertion via touch screen and showed that user performance significantly increases when s/he is alerted by haptic stimuli about unwanted double clicks and slips. 
Research has also led to new solutions to enhance interaction with mobile phones exploiting combinations of vibrotactile output and gesture recognition ([4] and [9]).

Differently from the mentioned studies, this work aims to facilitate blind users mobility. We evaluate how the usability of our accessible application (equipped with RFID-based spatial support) improves by integrating the multimodal interface with haptic capabilities. [8] also proposes the use of RFID tags to help the blind orient themselves, but is focused on navigation rather than environment disclosure. Another mobile system conceived for helping blind in public transportation scenarios is Ubibus [1]. With this system the user may use either a PDA (equipped with a WLAN interface) or a Bluetooth mobile phone. The main objective of this system is to help blind or partially blind people to take the public transport (e.g. it activates a stop request, it informs the bus driver to stop, it announces next stop, etc.). In Ubibus context-awareness is one way to guess the user's situation and to try to reduce inputs required from the user. In [5] an assistive system for the blind based on ubiquitous computing technology, called chatty environment, is proposed. The main idea behind the system is to enhance the visual information existing around us by other means of information that can be experienced by the visually impaired. The system provides useful information by exploiting Electronic markers used to tag the objects, captured by a mobile device in order to have descriptive information. Although these prototypes provide information on the surrounding environment, they do not offer a support to assist users in moving towards the tagged objects.

\section{THE ACCESSIBLE MOBILE GUIDE}

In order to allow blind users to freely move and access information related to nearby artworks and specimens, orientation support is particularly important. In our mobile guide we already integrated an electronic compass located in a small box hung on a cord round the user's neck, which is able to communicate with the guide software installed on the PDA through Bluetooth. The compass is lightweight and can be comfortably worn. The guide automatically detects the position of the available objects through RFID tags and a reader plugged into the PDA. In our solution, the tagged objects are detected when they are at about five meters' distance. The correspondence between artworks and tags is specified in the museum database. The position of each artwork within the rooms is stored in the database as well and is essential for suggesting the right direction to the users. In this paper, we present how we have extended such solution with vibrotactile feedback for orientation purposes. In this case, the haptic output modality is implemented through two separate actuators on the users' fingers to provide feedback on two opposite directions (left and right). Considering the context of our mobile guide, which is mostly an application for describing works of interest, we have limited the usage of haptic feedback to support the task of moving from one artwork to another. Section/artwork descriptions and notification messages are provided through a vocal user interface. An embedded TTS (Text To Speech) engine by Loquendo synthesizes the speech on the fly.

\subsection{VibroTactile Module}

The haptic output module, which is an add-on that we have specifically designed and tuned, consists of a plastic box (slightly thinner than a packet of cigarettes) fixed to the back of the PDA.
The box contains circuitry able to detect infrared signals. The photodiode (i.e. infrared receiver) protrudes from the box so that it is aligned to the infrared port of the PDA. The battery-operated circuitry also drives two vibrotactile actuators according to the commands sent by the PDA via the infrared interface. Each motor is attached to a rigid surface of about $1 \mathrm{~cm}^{2}$ and is connected to the box by a $10 \mathrm{~cm}$ wire. The motors can be fixed to the index finger and thumb by Velcro strips to let the rigid surfaces transmit vibrations to the fingertips. We opted for separating the motors from the box to facilitate distinguishing the channels. Otherwise, if motors were attached to the box it would have been very difficult to insulate them and the vibration of a single motor would have propagated to the rest of the device, making harder for the user to distinguish the vibrating side.

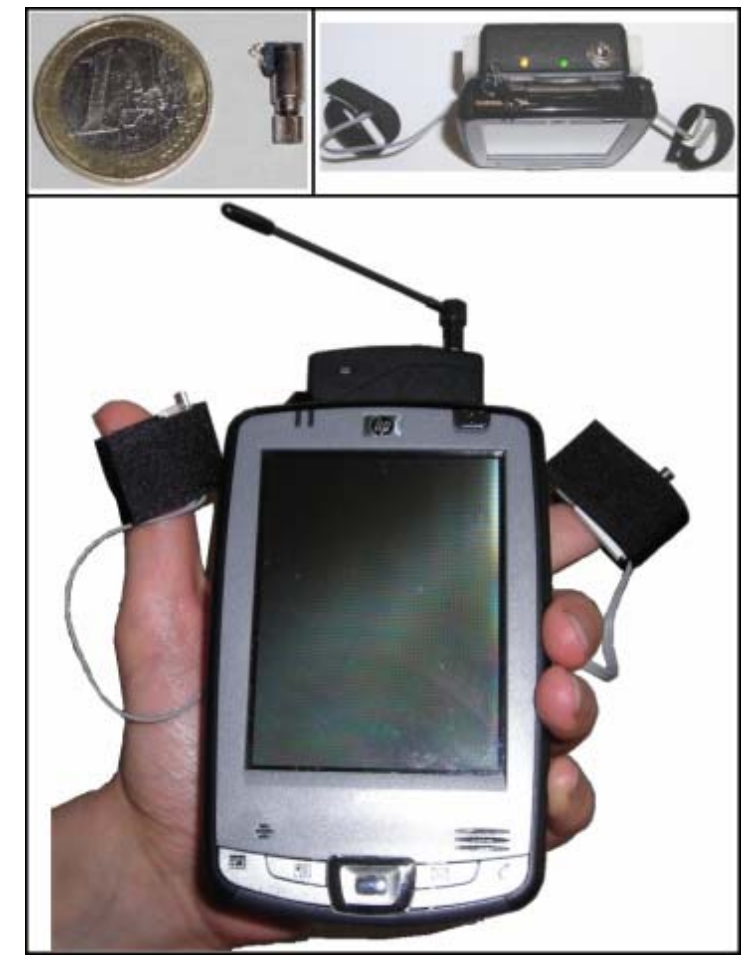

Figure 1. From the top left-hand corner: one of the actuators used in the experiment, the PDA fitted with the RFID reader and the haptic module, and how the device fits on the user's hand.

The actuators are RMV (Rotary Mass Vibrator) motors like those that enable vibration in many mobile phones (see Fig. [1] topleft). The driver circuitry controls each motor independently. Each command received from the PDA encodes the state of the haptic interface, that is, for each motor, the on/off flag and the vibration frequency value. The flag controls the switch (transistor) while the intensity value, a byte, is passed to a 256-step DPP (Digital Programmable Potentiometer). The haptic module circuitry is managed by a $4 \mathrm{MHz}$ microcontroller whose routine is able to decode a command and to upload the motor states up to 60 times per second.

To change the haptic device state the PDA software application has to generate and send a 3-bytes command with the switch flags, the intensity values and some check bits. For example, a 
haptic message like a short, intense left vibration requires two commands. The first, with left flag $=1$, right flag $=0$, left speed $=$ 255 (the maximum value) and right speed ignored initiates the left engine fast vibration. The second command, with left flag $=0$, right flag $=0$, left/right speed ignored stops the motor. The vibration length depends on the delay between the start and the stop commands. A complex haptic feedback such as a left-right fading can be created by repeatedly sending commands where the left speed parameter decreases and the right one increases (or vice versa). Since the latency between the sendCommand function call and the new motors configuration is about $15 \mathrm{~ms}$, we assume that even more complex effects may be created, such as the rhythms discussed in [3].

\subsection{Haptic Messages}

One of the aims of our study is to reduce the time and effort required to the blind user to move towards the next artwork. In facts, while localization is ensured by a grid of RFID tags and is provided by messages such as "you are approaching the artwork $x$ ", orientation (enabled by the electronic compass) is key support for successfully reaching the destination area.

In this application, usability is related to the time needed to reach the next artwork. Even in the smallest museums or exhibitions there are tens of artworks/items. The user should consider the guide as effective, i.e. as an alternative to the human companion. If not, the guide would be perceived as useless, turning the museum visit into a frustrating experience.

As already mentioned, haptic output has been investigated according to the suggestions made by some users of an earlier test. It has been considered as a complementary (rather than alternative) modality for signalling the best path to blind users.

We took into account that the museum visits are usually made just once by visitors and considered that they probably do not want to spend much time familiarizing themselves with the interface. For this reason, and taking into account the suggestions by users in a previous test, we configured haptic messages to encode only a small amount of information. We actually configured 4 types of messages corresponding to the vocal suggestions given by the previous version of the application: "Rotate Left", "Rotate Right", "Rotate Left a bit", "Rotate Right a bit". Rotation direction (left/right) is given by Spatial Location of the activated motor (i.e. which finger is vibrating). Rotation angle is indicated by Duration and Frequency of the vibration: a strong and long ( 2 seconds) impulse or a light and short $(700 \mathrm{~ms})$ one to indicate whether rotation must be more or less than $90^{\circ}$, respectively.

Haptic messages indicate the distance to the target as well: once the user has aligned to the best direction and has reached the destination area, short vibration pulses are activated on both sides. The delay between pulses reduces as the destination tag signal grows (i.e. the user approaches the tag). Feedback on the distance may help the user in centring the target even if the actually followed direction is slightly different from the ideal one.

\section{EVALUATION}

In order to evaluate our prototype we conducted a user test with a group of blind people. The evaluation concerned two versions of the guide that differ from the two ones previously tested [7] (which used for the orientation task one vocal feedback and one sound-based feedback). The previous versions adopted audio feedback based only on messages or sounds. The previous evaluation revealed that the vocal version using clear messages was preferred by the users. Furthermore, subjective opinions suggested that a vibrotactile feedback could have been an appropriate way to convey feedback. Thus, in this new evaluation we compared the version based on vocal messages alone with another new one using vibrotactile feedback for orientation. In the first version, tips such as "rotate left a bit" and repetitive "beep" sounds with variable delays were used to indicate distance and guide the user towards an artwork. The second version used right and left vibrotactile feedback to suggest direction and a double vibration corresponding to the repetitive "beeping" sounds with variable frequency to signal the distance. The users had to hold the PDA in the left hand and, for the vibrotactile version, put the two motors on the thumb and index finger (see Figure 1). The choice of the index finger rather than the middle finger is based on the sensitivity level [6].

\subsection{Method}

The goal of our test was to analyze whether there are some differences between the two different feedbacks. To this end, each user tried both versions to carry out the assigned tasks. An observational method was used to follow the users testing the prototypes. Through the conducted test both quantitative and qualitative data have been collected. First, in order to analyze the differences between the two versions, we measured the time taken by each user to carry out the tasks. Secondly, after the test each user was asked to fill in a questionnaire to gather subjective information and possible suggestions. The logged data were used to compare the effectiveness of the two versions. Indeed the time spent to perform each task has been used as a quantitative measure to compare the two types of feedback.

\subsubsection{Participants}

Eleven blind participants were recruited for the test: 7 women and 4 men. The age ranged from 27 to 66 years. All of them had used a screen reader in a Windows environment before. Six of them had previously used a PDA.

\subsubsection{Tasks}

Users were asked to reach a specific artwork by exploiting the compass-based guide. Because the experiment was mainly aimed at analyzing the kind of feedback, each user tried to reach different artworks by using the two versions of our prototype. In order to avoid a possible bias due to the learning process, five users used the version with vibrotactile feedback first and then afterwards the one based only on vocal feedback; whereas for the others the order was inverted. The task assigned was to start from a specific artwork to reach another one. The artworks to reach were different for the two guide versions. All users carried out the same tasks. Each test was carried out separately in order to avoid that users could be biased by observing other tests. We observed the users while they performed their tasks. As mentioned, the starting and finishing time was recorded in a log file for each user and for each task.

\subsection{Results}

All the users were able to accomplish the assigned tasks. The recorded time for each user for each task allowed us to compare the time spent by the user in carrying out the task when using the 
guide with the two different types of feedback. Compared time data revealed time saving in localizing the artwork by using the version with both vocal and vibrotactile support, though the difference was not statistically significant.

In order to evaluate the effective time saving we examined the data recorded for each user. The analysis was performed taking the users as the statistical unit and considering the time (s) taken by each user to accomplish the task.

Parametric analysis was applied according the two tests, Exact Kolmogov-Smirnov (N=11; $\mathrm{Z}=0.835-0.906 ; \mathrm{p}=\mathrm{ns})$ and Levene's test $(\mathrm{F}=1.179, \mathrm{p}=\mathrm{ns})$. A Paired $\mathrm{T}$ test $(\mathrm{N}=11, \mathrm{t}=0.463, \mathrm{df}=10, \mathrm{p}=$ $\mathrm{ns})$ revealed a non-significant difference in the time required to complete all tasks by using the two (vocal and vibrotactile) versions. This means that the two versions are similar and more or less equivalent for the purpose. Users declared that they felt more confident with clearer vocal messages, especially about the direction. Probably, in order to become more familiar with the vibrotactile feedback, it is necessary for blind users to experience it for a long period. Unfortunately, this is not the case, because our system is conceived for museum visitors who prefer short time for training.

Concerning subjective opinions, the users rated various aspects on a scale from 1 (the most negative value) to 5 (the most positive value). One aspect regarded the preference level for the two kinds of feedback. The expressed preferences revealed there is no significant difference between the two types of feedback. According to the Exact Kolmogov-Smirnov (N=11; $\mathrm{Z}=0.780$ $0.850 ; \mathrm{p}=\mathrm{ns})$ and Levene's test $(\mathrm{F}=0.508, \mathrm{p}=\mathrm{ns})$, we used a Paired T test, which revealed a non-significant difference $(\mathrm{N}=11$, $\mathrm{t}=1.047, \mathrm{df}=10, \mathrm{p}=\mathrm{ns})$. This result confirms the quantitative information gathered through the time saving. On average the users rated the vocal feedback well $(\mathrm{M}=3.81 ; \mathrm{SD}=0.98)$ and the vibrotactile version slightly less well $(\mathrm{M}=3.18 ; \mathrm{SD}=1.47)$. Various suggestions on how to improve both versions have been expressed through the questionnaires by the users. One stated that vibration intensity range should be made wider by increasing the motors' peak speed. Another would have preferred different vibration rhythms rather than intensities/durations to signal the angle of rotation. Two suggested increasing the updating frequency of tactile messages, in order to speed up the orientation process. Three users declared that an obstacle detection functionality would allow the avoidance of physical barriers, improving the autonomy of blind visitors.

\section{CONCLUSIONS AND FUTURE WORK}

In this paper we have presented a study conducted with eleven blind users to compare two versions of a mobile guide prototype. The conducted evaluation revealed that there is no significant difference between vocal and vibrotactile feedback. All users expressed a preference for one kind of feedback over the other. In particular, six users preferred the vibrotactile version, four the vocal one and only one assigned the maximum rating (5) to both versions. Quantitative analysis based on time saving in carrying out the tasks with the two versions confirmed these expressed preferences.

From this study we can conclude that both vibrotactile and vocal support could be appropriate in a mobile guide. Probably, a combination of both could represent a valid solution to improve the interaction. For example, when surroundings become noisy the vibrotactile feedback could be more appropriate. This is particularly true when the user may not want to disturb other people or to wear earphones. In a next version of our guide prototype we plan to add an obstacle sensor in order to further assist the users in the autonomous visit. In this perspective, vibrotactile feedback could be used for obstacle avoidance, and vocal messages for specific indications.

\section{ACKNOWLEDGMENTS}

Our thanks to Ivan Norscia for his suggestions in the statistical analysis of the test data and to the blind people who participated to our test.

\section{REFERENCES}

[1] Banâtre, M., Becus, M., Couderc, P. and Pauty, J. (2004). Ubibus: Ubiquitous Computing to Help Blind People in Public Transport. In the Proc. of Mobile Human-Computer Interaction (MobileHCI 2004), LNCS, Vol. 3160, pp. 310314.

[2] Brewster, S.A., Chohan, F.and Brown, L.M. (2007). Tactile Feedback for Mobile Interactions. In Proceedings of ACM CHI 2007 (San Jose, CA, USA), ACM Press AddisonWesley, pp 159-162.

[3] Brown, L.M., Brewster, S.A. and Purchase, H.C. (2006). Multidimensional Tactons for Non-Visual Information Display in Mobile Devices. In Proceedings of MobileHCI 2006 (Espoo, Finland), ACM Press, pp231-238.

[4] Brown, L.M. and Williamson, J. (2007). Shake2Talk: Multimodal Messaging for Interpersonal Communication. In Proceedings of HAID 2007, LNCS 4813, pp. 44-55, 2007

[5] Coroama, V. (2006). Experiences from the Design of a Ubiquitous Computing System for the Blind. In Proceedings CHI '06 Extended Abstracts on Human Factors in Computing Systems, pp. 664 - 669

[6] de Assis, F.M., Lima, A.C.O., Ramos de Souza, A.F., Silva e Silva, R.C. and Silverio Freire, R.C. (2007). Comparative Analysis of Tactile Sensibility between the Fingers in Vibrotactile Stimulus Recognition. In Proc. "Instrumentation and Measurement Technology Conference", 2007 IEEE, pp. 1-6.

[7] Ghiani, G., Leporini, B. and Paterno, F. (2008). Supporting orientation for blind people using museum guides. CHI'08 Extended Abstracts. ACM Press, pp. 3417-3422, DOI=http://doi.acm.org/10.1145/1358628.1358867.

[8] Helal, S., Willis, S., RFID Information Grid for Blind Navigation and Wayfinding. In Proceedings of the 9th IEEE Symposium on Wearable Computers (2005).

[9] Hughes, S., Murray-Smith, R. and Williamson, J. (2007). Shoogle: Multimodal Excitatory Interaction on Mobile Devices. In Proceedings of ACM SIG CHI 2007.

[10] Oakley, I., Kim, Y. and Ryu, J., (2006). Determining the Feasability of Forearm Mounted Vibrotactile Displays. In Proceedings of IEEE Haptics Symposium'06. 